\title{
Modeling of Field-Aligned Guided Echoes in the Plasmasphere
}

\author{
Shing F. Fung \\ Space Physics Data Facility \\ NASA/Goddard Space Flight Center \\ Greenbelt, MD \\ James L. Green \\ Space Science Data Operations Office \\ NASA/Goddard Space Flight Center \\ Greenbelt, MD
}

Submitted for Publication in the Journal Geophysical Research

June 29, 2004 


\begin{abstract}
The conditions under which high frequency $\left(f \gg>f_{\mathrm{uh}}\right)$ long-range extraordinary-mode discrete field-aligned echoes observed by the Radio Plasma Imager (RPI) on board the Imager for Magnetopause-to-Aurora Global Exploration (IMAGE) satellite in the plasmasphere are investigated by ray tracing modeling. Field-aligned discrete echoes are most commonly observed by RPI in the plasmasphere although they are also observed over the polar cap region. The plasmasphere field-aligned echoes appearing as multiple echo traces at different virtual ranges are attributed to signals reflected successively between conjugate hemispheres that propagate along or nearly along closed geomagnetic field lines. The ray tracing simulations show that fieldaligned ducts with as little as $1 \%$ density perturbations (depletions) and less than 10 wavelengths wide can guide nearly field-aligned propagating high frequency $\mathrm{X}$ mode waves. Effective guidance of wave at a given frequency and wave normal angle $(\Psi)$ depends on the cross-field density scale of the duct, such that ducts with stronger density depletions need to be wider in order to maintain the same gradient of refractive index across the magnetic field. While signal guidance by field aligned density gradient without ducting is possible only over the polar region, conjugate field-aligned echoes that have traversed through the equatorial region are most likely guided by ducting.
\end{abstract}

\title{
1. INTRODUCTION
}

It has long been shown that radio signals launched into ionospheric magnetic field-aligned density irregularities $(F A I)$ can be guided or ducted along magnetic field lines [e.g., Muldrew, 1963; 1967; Platt and Dyson, 1989; James, 2000;], analogous to whistler mode waves being guided along magnetic field lines in the magnetosphere (e.g., see Smith [1961], Helliwell [1965]). Ducting of freely propagating electromagnetic waves has also been expected to occur in the magnetosphere [Calvert, 1981;1995]. In the case of ducting, $F A I$ can act like a waveguide that traps and channels wave energy along the background magnetic field. For high-frequency electromagnetic waves $\left(f>>f_{\mathrm{uh}}\right.$, where $\mathrm{f}_{\mathrm{uh}}$ is the upper hybrid resonance frequency), a region of 
density depletion extending some distance along the magnetic field can trap wave energy by total internal reflections along a field-aligned density duct, as depicted in Figure 1.

In analyzing data taken by the Radio Plasma Imager (RPI) on board the Imager for Magnetopause-to-Aurora Global Exploration (IMAGE) satellite, Reinisch et al., [2001] suggested another possible signal guiding mechanism due to the presence of field-aligned density gradients, such as might be present over the polar cap regions. In this scenario, field-aligned propagating radio signals can naturally follow the magnetic field line without the trapping action of an electron density duct.

The RPI, the first instrument to perform long-range magnetospheric sounding successfully [Reinisch et al., 2000], transmits coded electromagnetic waves over a selectable frequency range from $3 \mathrm{kHz}$ to $3 \mathrm{MHz}$ from a long dipole antenna. The far-field radiation pattern of each transmitted pulse covers nearly all directions. From a sweep over a series of sounding frequencies RPI often observes return signals with discrete virtual-range and frequency characteristics over widespread ranges of altitudes and magnetic latitudes varying from the polar cap to the equator [Fung et al., 2002; 2003]. Figure 2a shows an RPI plasmagram (virtual range versus frequency) when the IMAGE spacecraft was just inside the plasmasphere. The virtual range is given by $\mathrm{ct} / 2$ where $\mathrm{c}$ is the free-space speed of light and $\mathrm{t}$ is the echo delay time. The echo intensity is color-coded. Three basic types of RPI echoes (field-aligned, diffuse, and resonance) are all recorded in Figure 2a. Plasma resonances observed by RPI and their usefulness in deriving local plasma and magnetic field strengths [e.g., Fung et al., 2003] have been discussed extensively by Benson et al. [2003]. The diffuse echoes typically show range or frequency spreading whereas field-aligned echoes tend to appear in discrete traces in plasmagrams and in many cases multiple traces over the same frequency range. Based on direction analysis and the virtual range determination the ray paths of the diffuse and fieldaligned echoes of Figure 2a are shown schematically in Figure 2b. Carpenter et al., [2002] and 
Fung et al [2003] attributed the range spreading of the diffuse echoes to coherent (aspectsensitive) scattering from field-aligned electron density irregularities $(F A I)$ having $<10 \%$ variations of the background density that range in size from $200 \mathrm{~m}$ to a few $\mathrm{km}$ as illustrated in Figure 2c, although Carpenter et al [2002] also pointed out the possibilities of refraction into and partial propagation along ducts having cross-field scales of several signal wavelengths.

As Fung et al [2003] pointed out, the diffuse echoes seen from outside the plasmapause and the field-aligned guided echoes seen inside the plasmapause represent two manifestations of the presence of FAI. Although it is not clear what processes can produce echo-guiding conditions that can extend from hemisphere to hemisphere, the widespread occurrences of field-aligned guided echoes clearly point to the existence of such conditions in the diverse environments of the magnetosphere and ionosphere.

Fung et al. [2003] showed that multiple traces of RPI discrete echoes of the type shown in Figure 2a, particularly the so-called epsilon signature, are most likely due tó ducted signals from conjugate hemispheres along closed field-aligned paths, analogous to the mechanism generally invoked to explain similar ionospheric observations. Results from modeling of ducted signals in the ionosphere have been reported by Muldrew $[1963 ; 1967 ; 1980]$ and by Platt and Dyson [1989] and analysis of direct bi-static observations have been performed by James [2000].

The purpose of this paper is to investigate the plasma conditions and wave characteristics necessary for high-frequency extraordinary mode waves $\left(f \gg>f_{\mathrm{uh}}\right)$ to be guided along the geomagnetic field over large magnetospheric distances. In order to determine the circumstances responsible for guiding long-range discrete echoes observed by the RPI, we have performed raytracing calculations within a model magnetosphere. Results will be compared to studies of ducting in the ionosphere [e.g., James, 2000]. Aside from the similar dependence of the ducting mechanism on the cross-field wave refractive index profile, much of the results presented here 
are not directly applicable to ducting of whistler mode waves, we will not attempt to compare our results with those of whistler ducts.

\section{RAY TRACING}

The computer code used for the ray tracing calculations in this study was originally developed in two dimensions by Shawhan [1967] and extended to three dimensions by Green [1984]. The code simultaneously solves the set of first-order differential equations for ray propagation in spherical coordinates developed by Haselgrove [1955] and Haselgrove and Haselgrove [1960]. The ray tracing technique uses the cold plasma theory [Stix, 1992] to determine the index of refraction and compute the ray paths by using geometric optics formulations. Light rays in the freely-propagating $L-O$ and $R-X$ modes are reflected when they encounter their respective wave cutoffs,

$$
\begin{aligned}
& f_{L O}=f_{p} \cong 9 \sqrt{N_{e}} \\
& f_{R X}=\frac{f_{g}}{2}+\left[\left(\frac{f_{g}}{2}\right)^{2}+f_{p}^{2}\right]^{1 / 2}
\end{aligned}
$$

where $f_{p}, f_{g}$, and $N_{e}$ are the electron plasma frequency, gyro frequency, and electron density, respectively. Thus the frequency of the reflected signals can be used to determine the local electron density at the cutoff.

To initiate a ray-tracing run, the code requires inputting a location of the source region (e.g., satellite position), wave frequency, the mode of propagation $(L-O$ or $R-X)$ and the initial wave normal angle (angle between the wave vector $k$ and the local magnetic field $B$ ) as initial conditions. Ray paths will then be calculated based on the propagation characteristics of a given mode in the model magnetosphere. Prior to the launch of the IMAGE spacecraft this code was 
used extensively to predict the traces of free space mode echoes [Fung and Green, 1996; Green et al., 2000]. According to Haselgrove [1955], the ray equations that describe the propagation of an electromagnetic wave in cold magnetized plasma are given by

$$
\begin{aligned}
& \frac{d r}{d t}=\frac{1}{n^{2}}\left(n_{r}-n \frac{\partial n}{\partial n_{r}}\right) \\
& \frac{d \theta}{d t}=\frac{1}{r n^{2}}\left(n_{\theta}-n \frac{\partial n}{\partial n_{\theta}}\right) \\
& \frac{d \phi}{d t}=\frac{1}{r n^{2} \sin \theta}\left(n_{\phi}-n \frac{\partial n}{\partial n_{\phi}}\right) \\
& \frac{d n_{r}}{d t}=\frac{1}{n}\left(\frac{\partial n}{\partial t}+n_{\theta} \frac{d \theta}{d t}+n_{\phi} \frac{d \phi}{d t} \sin \theta\right) \\
& \frac{d n_{\theta}}{d t}=\frac{1}{r}\left(\frac{1}{n} \frac{\partial n}{\partial \theta}-n_{\theta} \frac{d r}{d t}+r n_{\phi} \frac{d \phi}{d t} \cos \theta\right) \\
& \frac{d n_{\phi}}{d t}=\frac{1}{r \sin \theta}\left(\frac{1}{n} \frac{\partial n}{\partial \phi}-n_{\phi} \frac{d r}{d t} \sin \theta-r n_{\phi} \frac{d \theta}{d t} \cos \theta\right)
\end{aligned}
$$

where $n^{2}=\frac{c^{2}}{\omega^{2}} \vec{k} \cdot \vec{k}=n_{r}^{2}+n_{\theta}^{2}+n_{\phi}^{2}$ is the square of the index of refraction $n$ with $\vec{k}=k_{r} \hat{r}+k_{\theta} \hat{\theta}+k_{\phi} \hat{\phi}$ being the wave normal vector.

In order to simulate guided wave propagation in the plasmasphere, we have adopted a linear combination of several analytical models to prescribe the spatial variations of $n$. These include a diffusive equilibrium model (how the plasmas distributed) by Angerami and Thomas [1964], an ionosphere and plasmasphere compositional model ( $\%$ of electrons, protons, $\mathrm{He}^{+}$, and $\mathrm{O}^{+}$) by Kimura [1966], a plasmapause distribution (location and magnitude of the density gradient) by Aikyo and Ondoh [1971], a field-aligned density depleted duct (described below), and a dipole magnetic field model. These models provide the background plasma and structure of the plasmasphere over which our ray tracing simulations are performed. 
In order to simulate the effects of a field-aligned density duct on the RPI transmitted signals, a duct density model is added. The total electron density inside a field-aligned density duct is modeled by

$$
N(r, \theta)=N_{D E}\left\{1+\frac{\delta N}{N_{D E}} \exp \left[-\left(\frac{r-L \cos ^{2} \theta}{\sigma}\right)^{2}\right]\right\}
$$

where $N_{D E}$ is the diffusive equilibrium model density, $\delta \tilde{N} N_{D E}$ is the fractional density change at duct center, $\mathrm{r}$ is radial distance of a field location, $\sigma$ is the "width" of the density duct, and $\theta$ is the magnetic latitude of the field location at $r$. It is clear from equation (6) that the density perturbation maximizes at $\mathrm{r}=L \cos ^{2} \theta$, i.e., along a field line identified by the dipole $L$ value, corresponding to a maximum change in $N_{e}(\mathrm{x})$ across the background field (see Figure 1). For freely propagating modes, density depletions $(\delta N<0)$ in the duct lead to enhancements of the refractive index $n(\mathrm{x})$ at the duct center relative to the edges, such that total internal reflection within the duct is possible in accordance to Snell's law [Born and Wolf, 1999]. On the other hand for whistler propagation, refractive index enhancements are caused by density increases $(\delta N>$ 0 ), although whistler ray directions are already confined generally to within $20^{\circ}$ of the background magnetic field lines [Storey, 1953; Stix, 1992].

\section{RESULTS OF RAY TRACING CALCULATIONS}

As discussed above, RPI generates phase-encoded emissions at all wave normal angles at each sounding frequency. Using the models described above, ray paths of extraordinary-mode waves launched from a given position having different initial wave normal angles are calculated by solving equations (3) - (8). For simplicity, waves are launched from the field line at the center of the field-aligned density duct given by (9). Figure 3 shows $200-\mathrm{kHz} \mathrm{X}$-mode rays (blue and green traces) launched in the magnetic meridian plane with initial wave normal angles $\psi=0^{\circ}-360^{\circ}$, each separated from one another by $10^{\circ}$. The rays paths are launched from a 
point at $20^{\circ}$ magnetic latitude and $L=3.5$. The duct model density has a depletion $\delta \tilde{N} N_{\mathrm{DE}}=-$ $15 \%$ and $\sigma=20 \lambda($ or $30 \mathrm{~km})$.

Figure 3 shows that most rays with large wave normal angles escape the density duct and propagate into the plasmasphere. Rays that are launched obliquely to the magnetic field toward the interior of the plasmasphere are refracted and reflected at the right hand-extraordinary (R-X) cutoff surface, given by equation (2) and the red contours. Rays launched outward simply escape the plasmasphere. Only rays with relatively small wave normal angles (shown as green traces) are trapped and guided by the model density duct. Figure 4a shows a close-up view of nine 120$\mathrm{kHz}$ rays launched at different wave normal angles from $0^{\circ}$ to $72^{\circ}$ at every $8^{\circ}$ interval toward the northern hemisphere from the magnetic equator. Only rays with relatively small wave normal angles $(\psi<309$ are guided along the duct by total internal reflection and that only the ducted rays (green traces) can propagate to reach both conjugate hemispheres and are reflected back to the launching point, as shown in Figure $4 \mathrm{~b}$.

\subsection{EFFECT OF DUCT WIDTH}

In this section, we investigate how the effectiveness of ducting may depend on duct width, $\sigma$. For a given field-aligned density duct with a fixed depletion $\delta \tilde{N} N_{\mathrm{DE}}<0$, varying the duct width means changing the rate at which density increases from the center toward the "edge" of the duct $\left(\mid \mathrm{r}-L \cos ^{2} \theta>\sigma\right)$, where the background density level $N_{\mathrm{DE}}$ is recovered (see equation 9). For a given frequency wave propagating with a small but finite wave normal angle, the width of the duct also governs how quickly the wave encounters significant density effects toward the edge of the duct. Wave trapping (and guiding) by a field-aligned duct, as depicted in Figure 1, occurs when a wave propagating obliquely near the center of the duct is refracted so that the wave propagates toward the edge of the duct with an angle of incidence $\phi=\left|90^{\circ}-\psi\right|$ greater than the critical angle $\phi_{\mathrm{c}}$ for total internal reflection [Born and Wolf, 1999]. 
If the wave frequency far exceeds the local cutoff frequencies (equations 1 and 2), however, the wave will simply go through the ducting region and escape. On the other hand, waves with frequencies below the cutoff frequencies are evanescent and thus cannot propagate. Therefore a field-aligned density duct of a given depletion and width can only trap waves in limited frequency range, $1<f / f_{\mathrm{RX}}<\beta$. Since the density depletion is typically of order of a few percent, we can normalize the wave frequency $f$ by the local $R-X$ cutoff frequency (equation 2 ) based on $N_{\mathrm{DE}}$ and $f_{g}$. Similarly we can express duct width as $\sigma=\eta \lambda$, or $\tilde{\sigma}=(\sigma \lambda)=\eta$ with $\lambda$ being the freespace wavelength, and determine $\beta$ locally by launching rays at different positions along a duct.

Figure 5 shows the maximum ducted frequency $\beta=f_{\max } / f_{\mathrm{RX}}$ of waves at different $\psi$ as a function of normalized duct width $\eta$ for two different depletion levels. The figure indicates that higher frequency waves are more easily ducted when they are closer to field-aligned propagation and in deeper depletion ducts. For a fixed depletion, a gentle transverse density gradient associated with a wider duct thus can only trap lower frequency waves because high frequency waves with shorter wavelengths can escape the duct more easily. For all duct sizes (with $\sigma>$ few $\lambda$ ), all obliquely propagating waves trapped, say $\psi>10^{\circ}$, can only have frequencies slightly greater than the local $f_{\mathrm{RX}}$. Figure 6 shows the ray-tracing results of $\beta$ as a function of $\eta$ for different depletion levels when the initial wave normal angle is $0^{\circ}$, indicating the maximum ducted frequency ranges for different sets of duct parameters: $\delta \tilde{N} N_{\mathrm{DE}}$ and $\eta$.

As shown in Figure 5, waves at frequencies $f<10 f_{\mathrm{RX}}$ and have different wave normal angles can be guided by field aligned density ducts with $\delta \tilde{N} N_{\mathrm{DE}}<10 \%$. The echoes having slightly different directions of propagation will have different time delays, causing a small spread in virtual range or a finite width of the discrete echo trace. In order to understand the virtual range spreading effect due to different signal propagation directions, we have performed the ray tracing calculations shown in Figure 7. Assuming a duct with $\eta=50$ and $\delta \tilde{N} N_{\mathrm{DE}}<5 \%$, Figure 7 shows that a $0.2 \mathrm{R}_{\mathrm{E}}$ variation in virtual range can result from echoes $\left(f / f_{\mathrm{RX}}=1.1\right)$ having different initial 
wave normal angles $\left(0^{\circ}<\psi<18^{\circ}\right)$, enough to explain most typical widths of discrete (guided) echo traces observed by RPI in the magnetosphere [Fung et al., 2003]. Larger range spreading ( $0.5 \mathrm{R}_{\mathrm{E}}$ ), such as reported by Carpenter et al. [2002] and Fung et al [2003], may be caused by coherent scattering processes.

\subsection{SIMULATED PLASMAGRAM}

We can use ray-tracing modeling to construct simulated plasmagrams and show that observed plasmagram traces may in fact result from ducting. Figure 8 shows the results of ray tracing simulated plasmagrams when ducted rays are launched at the equator (panel A) and at $20^{\circ}$ magnetic latitude (panel B). With only a few percent density depletion $\left(\delta \tilde{N} N_{\mathrm{DE}} \sim 5 \%\right)$ for the entire density duct, waves at frequencies that exceed slightly the local $R-X$ cutoff (equation 2 ) have relatively slow group speeds and thus have relatively long delay times or virtual ranges, causing the nearly-vertical portion of the trace as shown in panel A. Higher frequency waves with group speeds approaching light speed can reach deeper in the ionosphere where the density gradients are sharper, leading to the nearly flat or asymptotic trace appearance.

From a source at $20^{\circ}$ magnetic latitude within the model plasmasphere two distinct echo traces are observed in Figure 8B. The bottom trace of Figure 8B are simulated echoes from along the field line in the hemisphere closest to the Earth while the upper trace is from the conjugate hemisphere. The simulated echoes in Figure $8 \mathrm{~B}$ can be compared with the observed traces in Figure $2 \mathrm{~A}$ and show the excellent qualitative agreement between the corresponding local hemisphere (A) and conjugate-hemisphere (B) traces.

\section{SIGNAL GUIDANCE MECHANISM}

As mentioned earlier, Reinisch et al., [2001] suggested another possible mechanism to produce field-aligned echoes. In that scenario, field-aligned propagating radio signals would naturally follow the magnetic field line when the density (refractive index) gradient direction is parallel to 
the geomagnetic field line. This condition may be likely at high latitudes where the field lines and density gradients are more or less in the radial direction as discussed in Fung et al. [2003]. Inversions of multiple plasmagrams of field-aligned echoes during passages at high latitudes have allowed Nsumei et al [2003] to determine the polar cap densities under different geomagnetic conditions. As also pointed out in Fung et al [2003], however, the density gradients near the equator are mostly oblique or perpendicular to the magnetic field directions. This would make the density gradient unlikely to be the responsible guiding mechanism for guiding signals to and from the conjugate hemisphere.

Figure 9 shows quantitatively the angles between the model density gradients and the model local geomagnetic field lines as a function of magnetic latitude along the $L=2,2.5,3,3.5$ and 4 dipole field lines. As shown in Figure 9, the electron density gradients in the plasmasphere $(L \leq$ 4) become highly oblique to the geomagnetic field lines as the magnetic latitudes are less than $\sim 35^{\circ}$, indicating that signal guidance by field-aligned density-gradient is an unlikely mechanism that would lead to the discrete echoes (especially the ones from conjugate hemisphere) often observed by RPI in the plasmasphere. On the other hand, the density gradients (thus refractive index gradients) at sufficiently high $L$ values, e.g., over the polar cap, are more easily to become field-aligned.

The diffuse echoes, as shown in Figure 2a, that often accompany the field-aligned echoes observed in RPI plasmagrams can be viewed as corroborating evidence for the presence of fieldaligned density irregularities. Carpenter et al., [2002] and Fung et al [2003] attributed the range spreading of the diffuse echoes at least in part to coherent scattering from cross-field electron density irregularities of $<10 \%$ of the background density that range in size from $200 \mathrm{~m}$ to greater than a few $\mathrm{km}$. The ray tracing calculations in this paper clearly demonstrates that under these conditions we would expect to observe ducted echoes. 


\section{DISCUSSION}

Because of the rather extensive history in investigations of ionospheric ducts, it may be of interest to compare the duct density model employed here [equation (6)] and those use in ionospheric studies [e.g., James, 2000]. Equation (6) gives the cross-field electron density profile of a magnetospheric density duct along a dipole geomagnetic field line. The density structure along the field line length is assumed to be in diffusive equilibrium. The analytic prescription is used to facilitate the ray-tracing calculations presented earlier. As shown by equation (6), both the total density perturbation $\left(\delta \tilde{N} N_{\mathrm{DE}}\right)$ and the duct width $\sigma$ determine the cross-field density gradient of the duct, which controls the amount of refraction imposed by the duct on a given propagating wave $(f, \psi)$. Although a constant $\sigma$ is used in the present study, the changing $N_{D E}$ along the magnetic field actually provides a relatively realistic treatment of the physical situation. An extension of the model is to incorporate a $\sigma$ that varies with distances along the field, but we expect those results to be essentially the same as here.

In a recent study of ionospheric ducts using bi-static radio observation, James [2000] used a cylindrical duct model with exponentially decreasing electron density with altitude. The crosssectional duct density profile is modeled by a form factor given by $\left\{1-\cos \left[\pi\left(r / r_{D}\right)^{m}\right]\right\}$, where $r$ is the radial distance from the duct center, $\mathrm{r}_{\mathrm{D}}$ is the cylindrical duct radius, and $\mathrm{m}$ specifies the steepness distribution of the cross-field density gradients. Unlike equation (6), this model is valid only for modeling field-aligned density ducts in limited altitude range in the low-altitude polar ionosphere with fixed scale height and essentially vertical (radial) field-line geometry.

The Gaussian cross-field density profile in equation (6) is known to be a "well-behave" function with no sharp turns that seems to lead readily to ducting of waves with wave-normal directions less than $\sim 30^{\circ}$ from the magnetic field orientation (see Figure 3). On the other hand, the James [2000] model tends to require relatively steep duct walls $(m=4$ and $m=6)$ to trap waves even at 
a few degrees from the magnetic field. The model predicts no effective wave ducting for gentle sloping $(\mathrm{m}=1)$ of density or obliquely propagating waves (e.g., $\psi>$ few degrees).

We should also examine the conditions valid for ducting by $F A I$ in some details. As shown in Figure 10, radio signals is expected to be trapped by field-aligned density structures in which the refractive index $n(\rho)$ encountered by the wave is a decreasing function of the distance $\rho$ (measured normal to B) from the duct center. This occurs for high-frequency $R-X$ and $L-O$ mode waves when $\delta \tilde{N} N_{\mathrm{DE}}<0$, and for whistlers when $\delta \tilde{N} N_{\mathrm{DE}}>0$. As the wave propagates from a region of higher $n\left(\rho_{1}\right)$ (e.g., at points $\mathrm{A}, \mathrm{C}, \mathrm{E}$, and $\mathrm{G}$ ) to a region of lower $n\left(\rho_{2}\right)$ (at points $\mathrm{D}, \mathrm{H}$, $\mathrm{B}$, and $\mathrm{F}$ ), refraction of the wave would turn the wave propagation directions from more oblique to more parallel to $\mathbf{B}$. Total internal refraction or trapping of the wave occurs when the wave normal angle satisfies $\psi<\left(90^{\circ}-\phi_{c}\right)$, where $\phi_{c}$ is the critical incidence angle at which the refracted wave becomes parallel to the density duct or the background magnetic field. For proper ducting, the field-aligned density duct must also satisfy following conditions:

(1) Reflecting surfaces (shaded regions in Figure 10) along the length of the duct must be sufficiently smooth that they are all specular surfaces, i.e., the amplitudes of any surface roughness must be small compared to the wavelengths of the ducted waves. Otherwise, refraction and scattering by surface roughness could easily prevent ducting over long distances.

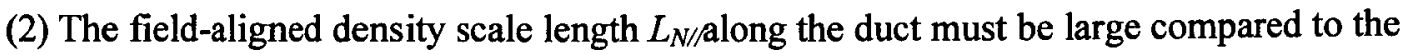
duct width $\sigma$, which is the cross-field scale over which $N_{e}$ may vary by a few percent. Furthermore, we must also have $\lambda<\sigma\left(\ll L_{N / /}\right)$ for wave propagation. Thus for a given duct width $\sigma$, there is a minimum wave frequency $f_{m} \sim \mathrm{c} / \sigma$ below which no waves can be ducted.

Based on conditions (1) and (2) above, we can estimate the smoothness and width of the density duct that may be responsible for the discrete echoes shown in Figure 2a. It is noticed that the discrete traces associated with the local hemisphere (trace A) and that reflected in the 
conjugate hemisphere (trace B) have low-frequency limits at $269.8 \mathrm{kHz}$ and $280.6 \mathrm{kHz}$, respectively. The low-frequency limit of trace $\mathrm{A}$ is observed with very small virtual range $(\sim 0.3$ $\mathrm{R}_{\mathrm{E}}$ ) and therefore must be near the local $R-X$ cutoff frequency at the spacecraft with a free-space wavelength $\lambda \sim 1.1 \mathrm{~km}$, which is also the limit of surface roughness along the density duct in the local hemisphere. The slightly higher low-frequency limit $(280.6 \mathrm{kHz})$ of the conjugate trace (B), however, suggests that signal guidance by the conjugate portion of the density duct is not so effective at lower frequencies perhaps due to surface roughness of the order of $1 \mathrm{~km} \mathrm{scale.} \mathrm{Both}$ traces $\mathrm{A}$ and $\mathrm{B}$ have a maximum frequency $f_{\mathrm{Max}}$ of $369.2 \mathrm{kHz}$ (with $\lambda \sim 0.81 \mathrm{~km}$ ), thus we have $f_{\mathrm{Max}} / f_{\mathrm{RX}} \sim 1.37$. This corresponds to a minimum duct size $\sigma \sim 10 \lambda_{\mathrm{Max}} \geq 81 \mathrm{~km}$ (for $1 \%$ depletion, see Figure 6).

\section{CONCLUSIONS}

The ray tracing calculations presented in this paper show that field-aligned density depletions in the plasmasphere could lead to ducting of high-frequency extraordinary mode waves (Figures 3 and 4). As.shown in Figures 5 and 6, proper guidance of wave at a given frequency $\left(f_{\mathrm{Max}} / f_{\mathrm{RX}}\right)$ and wave normal angle $\left(0<\psi<30^{\circ}\right)$ requires the duct width to increase with increasing density depletion $\left(\delta \tilde{N} N_{\mathrm{DE}}\right)$. Ducting of nearly field-aligned propagating waves $\left(\psi \sim 0^{\circ}\right)$ can occur within density depletions as small as $1 \%$ and less than 10 wavelengths wide (Figure 6). When launched at high altitudes, only nearly field-aligned propagating rays can be trapped and guided.

Conjugate field-aligned echoes observed by RPI are most likely due to waveguide ducting with relatively smooth ducts, as prescribed by conditions (1) and (2) above, maintained along much of the plasmaspheric field lines.

Acknowledgments. The authors would like to acknowledge V. Jayanti for programming assistance and implementation of the density duct model into the J. Green's ray tracing program. In addition the authors also acknowledge S. Boardsen for producing Figure 9 and discussions 
with X. Huang. The authors also benefited from comments on an earlier draft of the paper by R. F. Benson and D. L. Carpenter. This work was performed under UPN 51-370-28-30.

\section{REFERENCES}

Angerami, J. J., and J. O. Thomas, The distribution of ions and electrons in the Earth's exosphere, J. Geophys. Res., 69, 4537, 1964.

Aikyo, K., and T. Ondoh, Propagation of nonducted VLF waves in the vicinity of the plasmapause, J.. Radio Res. Labs., 18, 153, 1971.

Benson, R.F., V. A. Osherovich, J. Fainberg, and B. W. Reinisch, Classification of IMAGE/RPIstimulated plasma resonances for the accurate determination of magnetospheric electrondensity and magnetic-field values, J. Geophys. Res., 108 (A5), 1207, doi:10.1029/2002JA009589, 2003.

Born, Max and Emil Wolf, Principles of Optics, Cambridge University Press, p. 49, 1999.

Calvert, W., The detectability of ducted echoes in the magnetosphere, J. Geophys. Res., 86, 1609-1612, 1981.

Calvert, W., Wave ducting in different wave modes, J. Geophys. Res., 100, 17491-17497, 1995.

Carpenter, D. L., M. Spasojevic, T. F. Bell, U. S. Inan, B. W. Reinisch, I. A. Galkin, R. F. Benson, J. L. Green, S. F. Fung, S. A. Boardsen, Small-scale field-aligned plasmaspheric density structures inferred from RPI on IMAGE, J. Geophys. Res., 107(A9), 1258, 10.1029/2001JA009199, 2002.

Fung, S. F., and J. L. Green, Global imaging and radio remote sensing of the magnetosphere, Radiation belts Models and Standards, Geophysical Monogr., 97, AGU, Washington, D. C., 285-290, 1996.

Fung, S. F., R. F. Benson, J. L. Green, B. W. Reinisch, D. M.Haines, I. A. Galkin, J.-L. Bougeret, R. Manning, P. H. Reiff, D. L. Gallagher, D. L. Carpenter, and W. W. L. Taylor, 
"Observations of Magnetospheric Plasmas by the Radio Plasma Imager on the IMAGE Mission," Advances in Space Research, vol. 30, pp. 2259-2266, 2002.

Fung, S. F., R. F. Benson, D. L. Carpenter, J. L. Green, V. Jayanti, I. A. Galkan, and B. W. Reinisch, Guided Echoes in the Magnetosphere: Observations by Radio Plasma Imager on IMAGE, Geophys. Res. Lett., 30(11), 1589, doi:10.1029/2002GL016531, 2003.

Green, J. L., The Io decametric emission cone, Radio Science, 19, 556, 1984.

Green, J. L., R. F. Benson, S. F. Fung, W. W. L. Taylor, S. A. Boardsen, B. W. Reinisch, D. M. Haines, K. Bibl, G. Cheney, I. A. Galkin , X. Huang, S. H. Myers, G. S. Sales, J.-L. Bougeret, R. Manning, N. Meyer-Vernet, M. Moncuquet, D. L. Carpenter, D. L. Gallagher, and P. Reiff, Radio Plasma Imager measurements, Space Science Reviews, 91, 361-389, February, 2000.

Haselgrove, J., Ray theory and a new method for ray tracing, London Phys. Soc., Report of Conference on the Physics of the Ionosphere, 355, 1955.

Haselgrove, C. B., and J. Haselgrove, Twisted ray paths in the ionosphere, Proc. Roy. Soc. London, 75, 357, 1960.

Helliwell, R. A., Whistlers and Related Ionospheric Phenomena, Stanford University Press, Stanford, California, 1965.

James, G. H., Ionospheric duct parameters from a dual-payload radio-frequency sounder, $J$. Geophys. Res., 105, 20909-20918, 2000.

Kimura, I., Effects of ions on whistler-mode ray tracing, Radio Sci., 1, 269-283. 1966.

Muldrew, D. B., Radio propagation along magnetic field-aligned sheets of ionization observed by the Alouette topside sounder, J. Geophys. Res., 68, 5355-5370, 1963.

Muldrew, D. B., Medium frequency conjugate echoes observed on topside-sounder data, Can. J. Phys, 45, 3935-3944, 1967.

Muldrew, D. B., The formation of ducts and spread $F$ and the initiation of bubbles by field aligned currents, J. Geophys. Res., 85, 613-625, 1980. 
Nsumei, P. A., X. Huang, B. W. Reinisch, P. Song, V. M. Vasyliunas, J. L. Green, S. F. Fung, R. F. Benson, and D. L. Gallagher, Electron density distribution over the northern polar region deduced from IMAGE/radio plasma imager sounding, J. Geophys. Res., 108(A2), 1078, doi:10.1029/2002JA009616, 2003.

Platt, I. G., and P. L. Dyson, MF and HF propagation characteristics of ionospheric ducts, $J$. Atmos. Terres. Phys., 51, 759-774, 1989.

Reinisch, B. W., D. M. Haines, K. Bibl, G. Cheney, I. A. Galkan, X. Huang, S. H. Myers, G. S. Sales, R. F. Benson, S. F. Fung, J. L. Green, W. W. L. Taylor, J.-L. Bougeret, R. Manning, N. Meyer-Vernet, M. Moncuquet, D. L. Carpenter, D. L. Gallagher, and P. H. Reiff, The Radio Plasma Imager investigation on the IMAGE spacecraft, Space Science Reviews special issue on the IMAGE mission, 91, 319-359, 2000.

Reinisch, B. W., X. Huang, P. Song, G. S. Sales, S. F. Fung, J. L. Green, D. L. Gallagher, and V. M. Vasyliunas, Plasma density distribution along the magnetospheric field: RPI observations from IMAGE, Geophys. Res, Lett., 28, 4521-4524, December 15, 2001.

Shawhan, S.D., A computer program for VLF ray tracing in a model ionosphere, Research Report 67-12, University of Iowa, Iowa City, Iowa, 1967.

Smith, R. L., Propagation characteristics of whistlers trapped in field-aligned columns of enhanced ionization, J. Geophys. Res., 66, 3699-3707, 1961.

Stix, T. H., Waves in Plasmas, American Institute of Physics, New York, pp.26-17, 1992.

Storey, L. R. O., An investigation of whistling atmospherics, Phil. Trans. Roy. Soc. London, Ser. A $246,113,1953$. 


\section{Figure Captions}

Figure 1. A schematic of ducting of a high-frequency $\left(f>>f_{\text {uh }}\right.$ ) electromagnetic wave (green arrows) by field-aligned electron density depletions.

Figure 2. Panel A shows a typical RPI plasmagram with field-aligned and diffuse echoes labeled. Panel B illustrates the directions of the observed echoes relative to the spacecraft position depicted by the red dot. As shown in panel A, the diffuse echoes are spread in range and are therefore reflecting from a series of irregularities as shown in panel $\mathrm{C}$.

Figure 3. R-X mode rays at $200-\mathrm{kHz}$ with $\psi=0-360^{\circ}$ in $10^{\circ}$ intervals, launched at $20^{\circ}$ magnetic latitude and $\mathrm{L}=3.5, \delta \mathrm{N} / \mathrm{N}_{\mathrm{DE}}=-15 \%, \sigma=30 \mathrm{~km}$. Only rays with initial $\psi<30^{\circ}$ or with $\psi>150^{\circ}$ are guided by the model density duct.

Figure 4. Ducted and non-ducted ray at $f=120 \mathrm{kHz}, \delta \psi=8^{\circ}, 0^{\circ}$ magnetic latitude, $L=$ 3.5, $\delta N / N_{D E}=-5 \%, \sigma=150 \mathrm{~km}(60 \lambda)$ having different wave normal angles $\psi$ from $0^{\circ}$ to $72^{\circ}$ at $8^{\circ}$ intervals launched from the magnetic equator toward the northern hemisphere. Rays with large wave normal angles $(\psi>309)$ are not ducted.

Figure 5. Maximum ducted frequency $\beta=f_{\max } / f_{\mathrm{RX}}$ of waves (with different initial wave normal angles $\psi$ ) as a function of normalized duct width $\eta$ for a $2 \% \mathrm{~N}_{\mathrm{e}}$ depletion (panel A) and a $10 \%$ $\mathrm{N}_{\mathrm{e}}$ depletion (panel B). Larger density depletions can support sounder waves at progressively higher frequencies.

Figure 6. Variation of maximum ducted frequency $\beta=f_{\mathrm{MAX}} / f_{\mathrm{RX}}$ as a function of duct width for $0^{\circ}$ propagation in ducts with different $\mathrm{N}_{\mathrm{e}}$ depletions. 
Figure 7. Variation of virtual ranges of echoes at $f / f_{\mathrm{RX}}=1.1$ and different initial wave normal angles guided by a field-aligned density duct with $\eta=50$ and $\delta \tilde{N} N_{\mathrm{DE}}<5 \%$.

Figure 8. Simulated plasmagram traces when sounding from the equator (panel A) and from $20^{\circ}$ magnetic latitude (panel $\mathrm{B}$ ) in a field-aligned density duct with $5 \%$ depletion and $\eta=50$.

Figure 9. A plot of the angle between the model density gradient $(\nabla \mathbf{N})$ and the geomagnetic field line (B) along the $L=2,2.5,3,3.5$, and 4 field lines as a function of magnetic latitude.

Figure 10. A schematic showing the guidance of signals by total internal reflections along a density duct, where the refractive index $n$ as seen by the wave along the duct is a decreasing function of the distance from duct center. As the wave propagates along the duct, refraction of the wave changes the wave normal angle $\Psi$ from a finite value near the duct center to $0^{\circ}$ (for parallel propagation) or $180^{\circ}$ (for anti-parallel propagation) near the edge of the duct. 


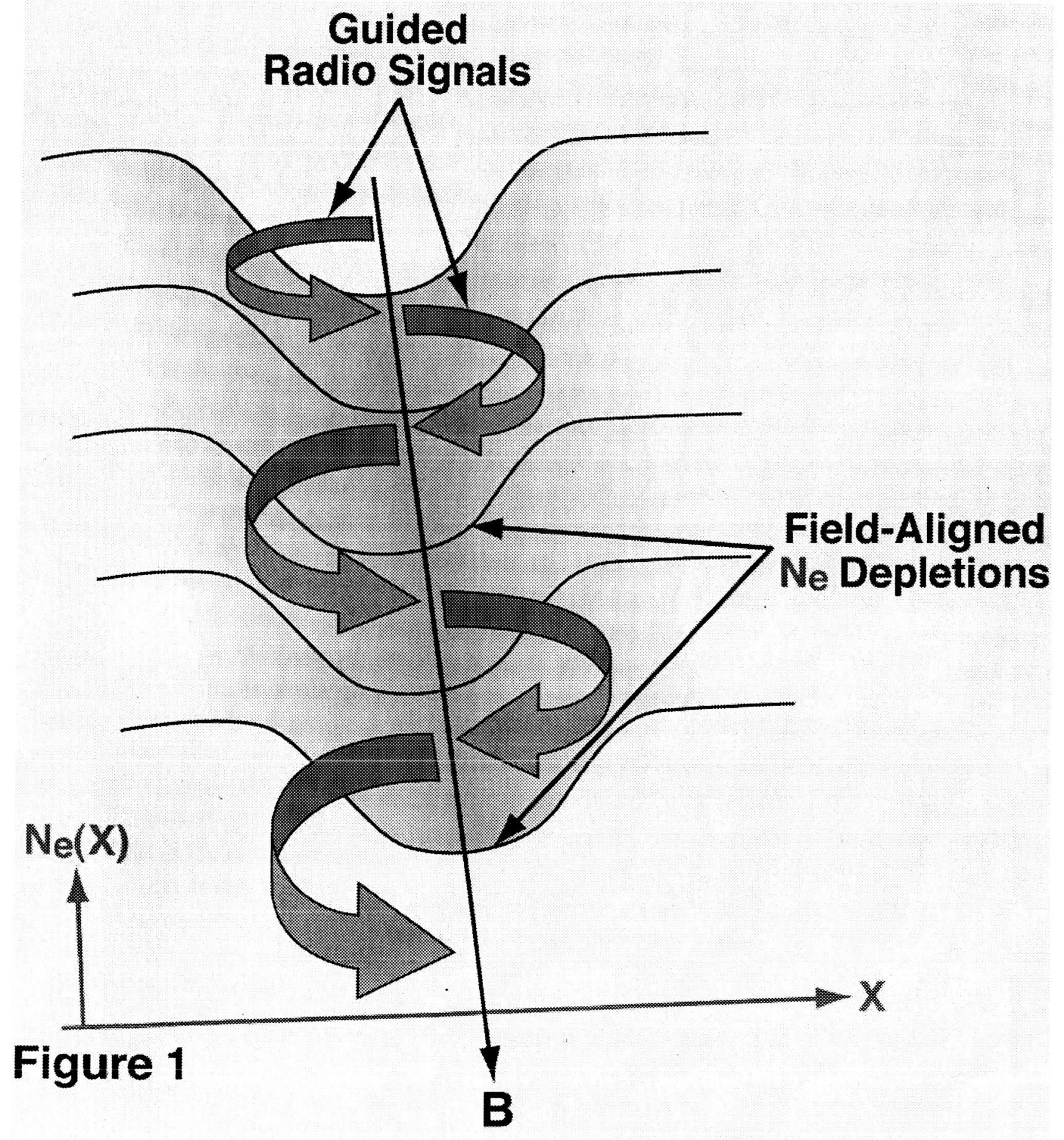



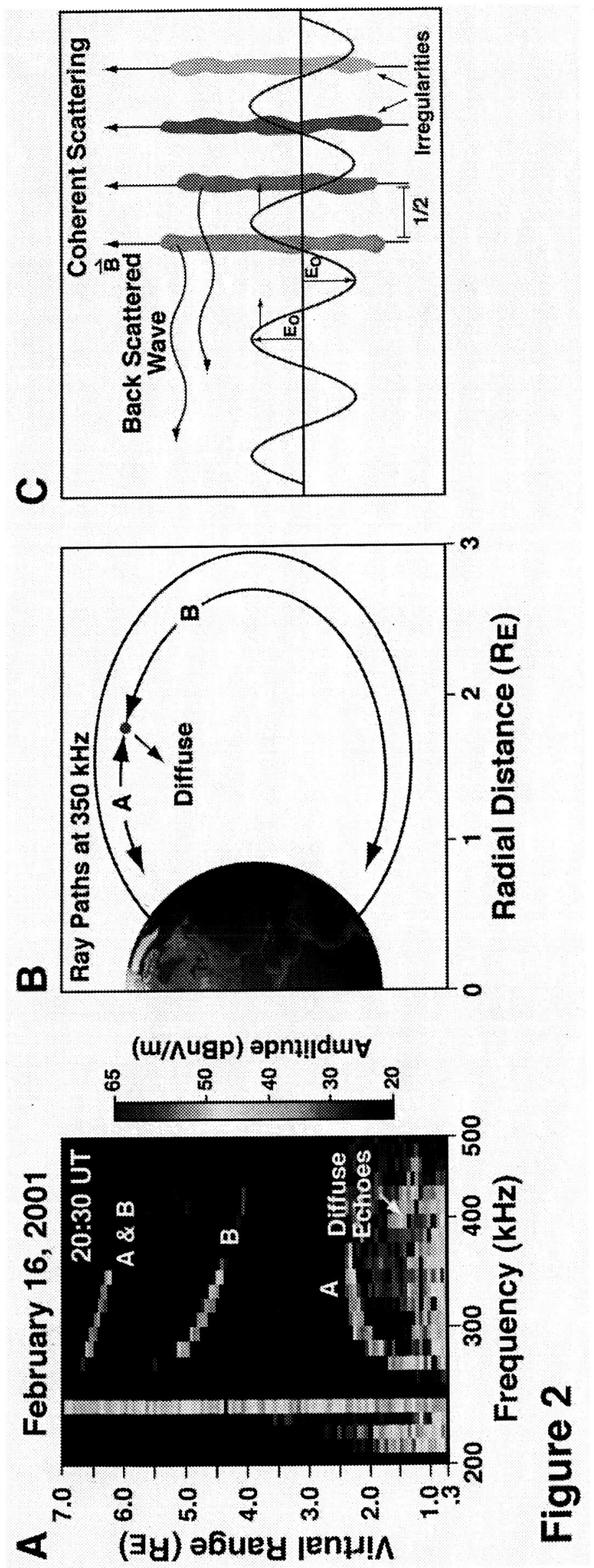


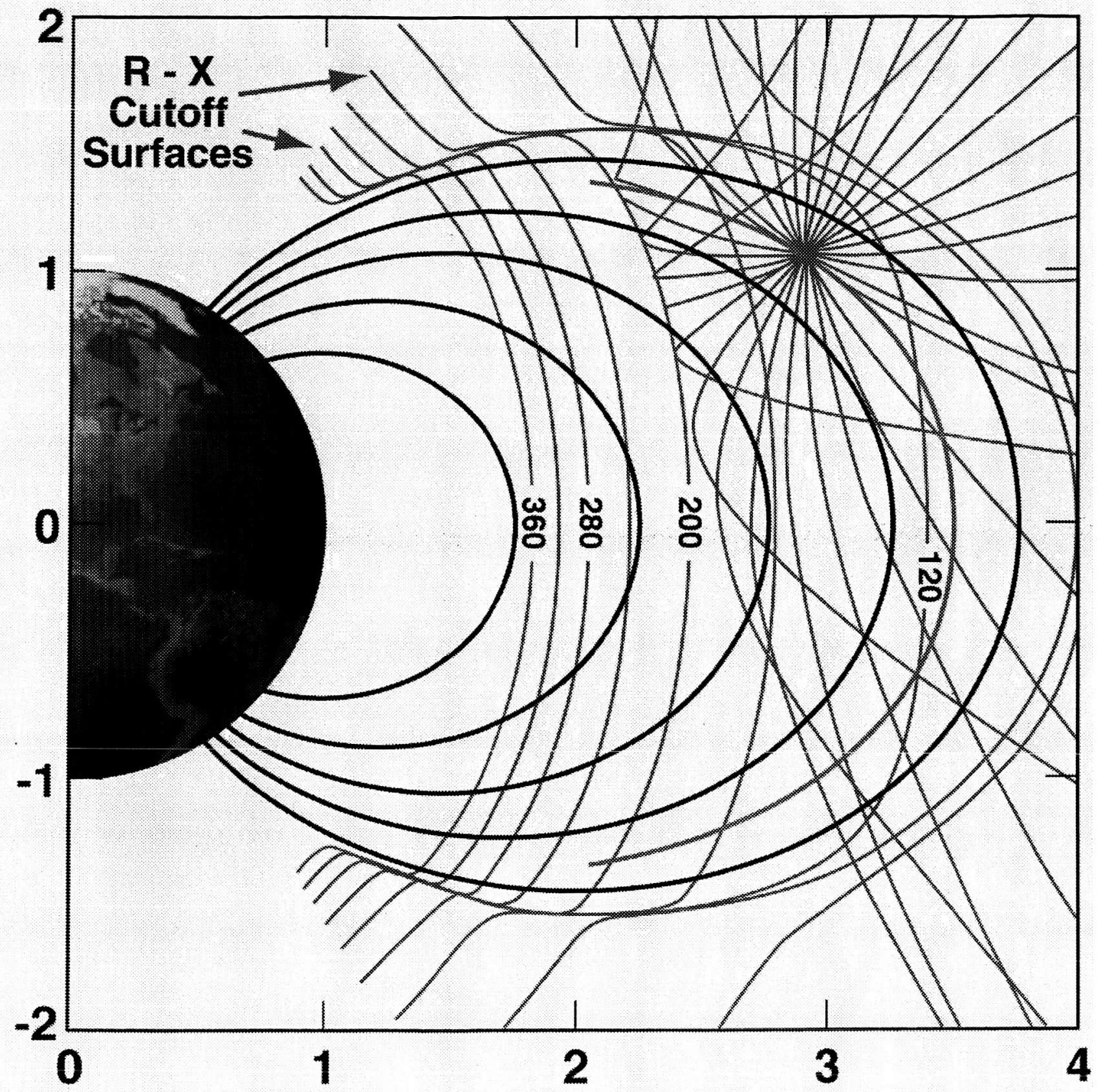

Figure 3 

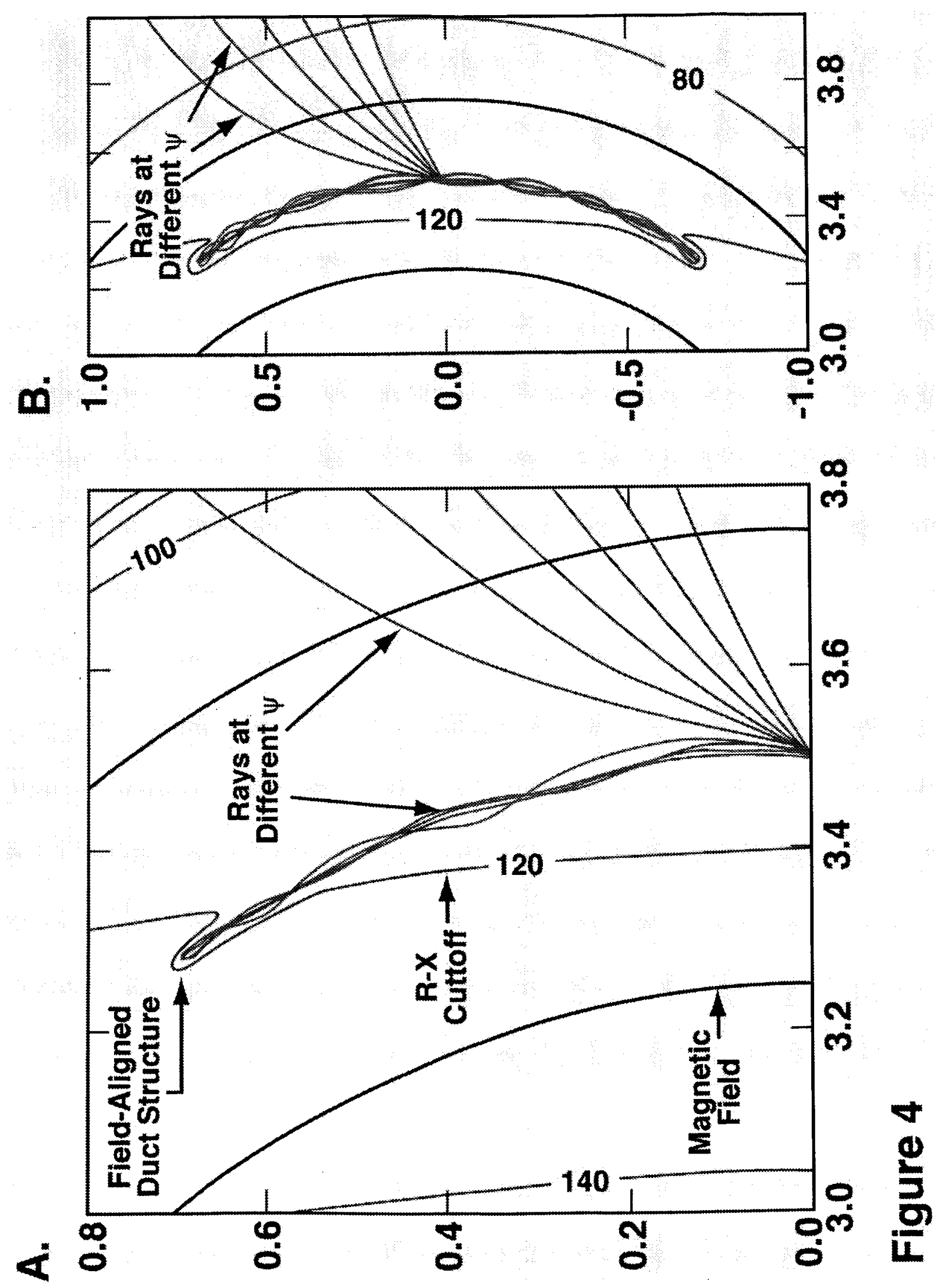

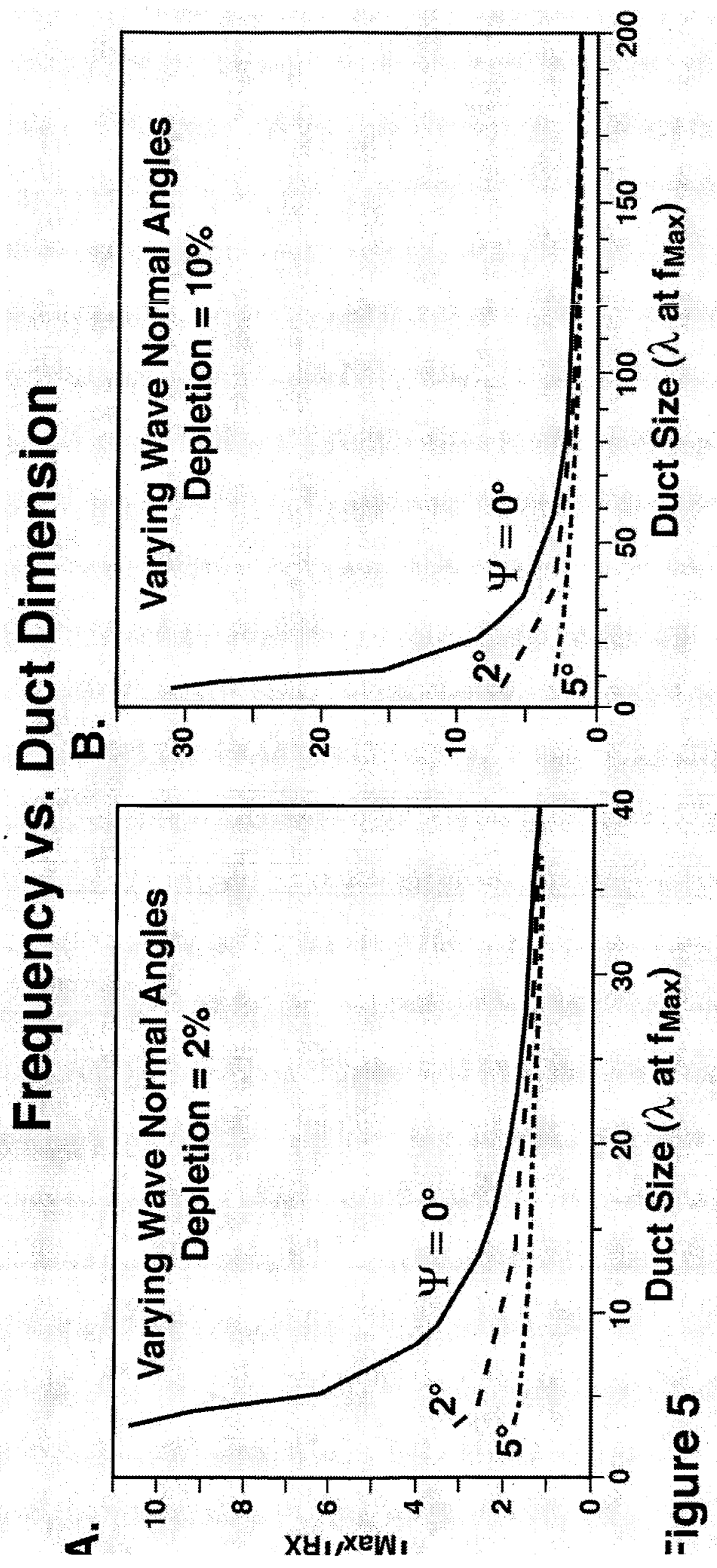


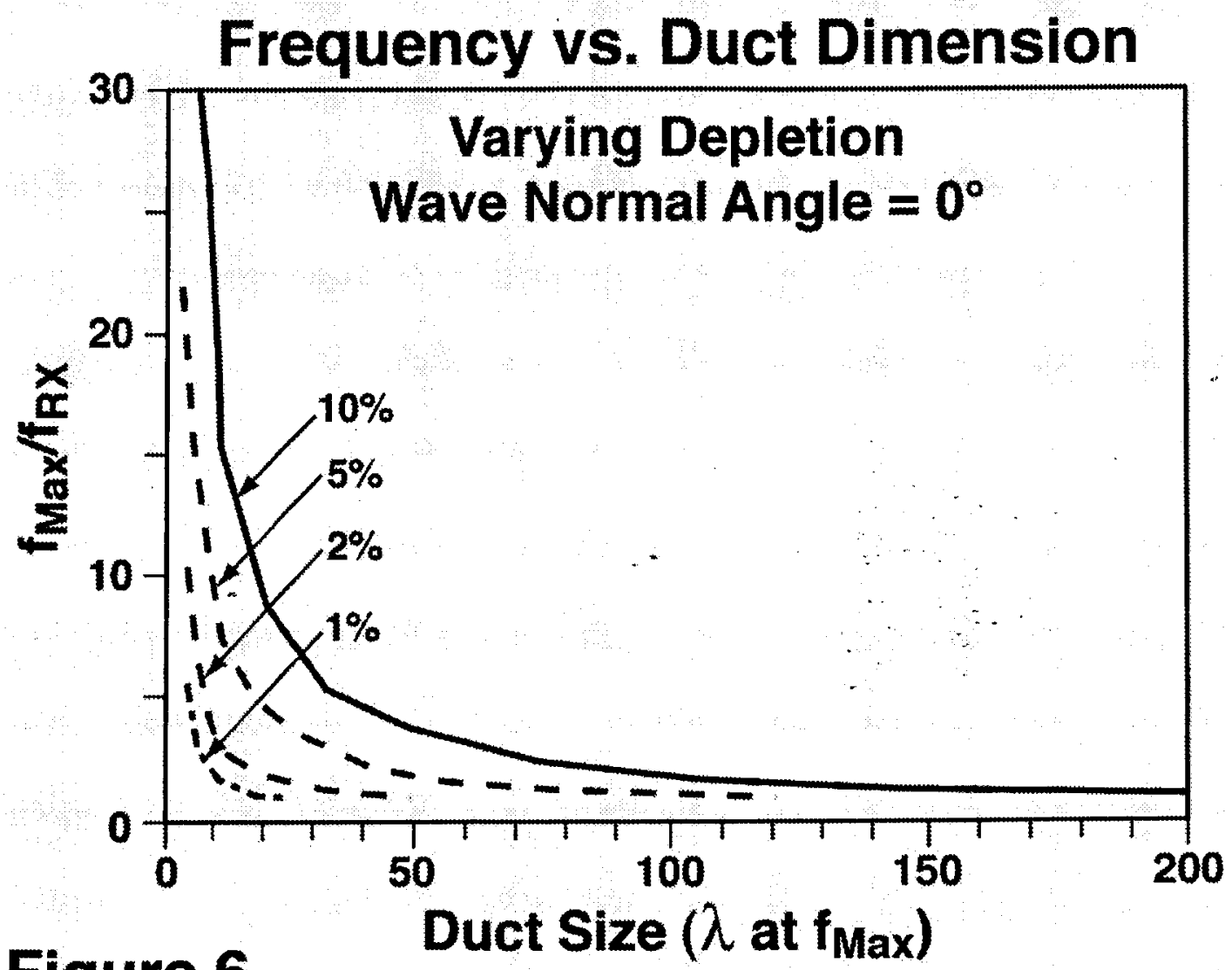

Figure 6 


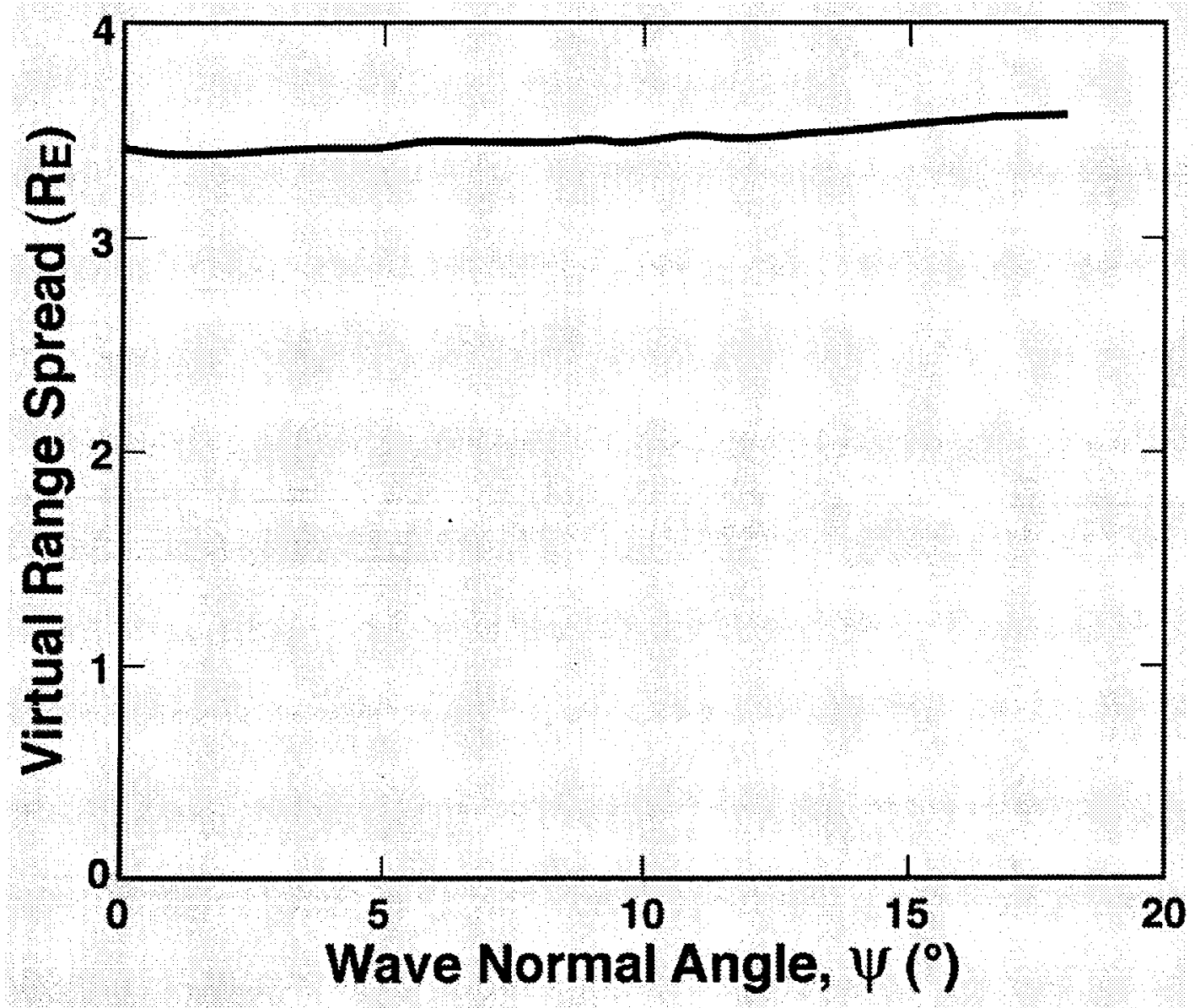

Figure 7 


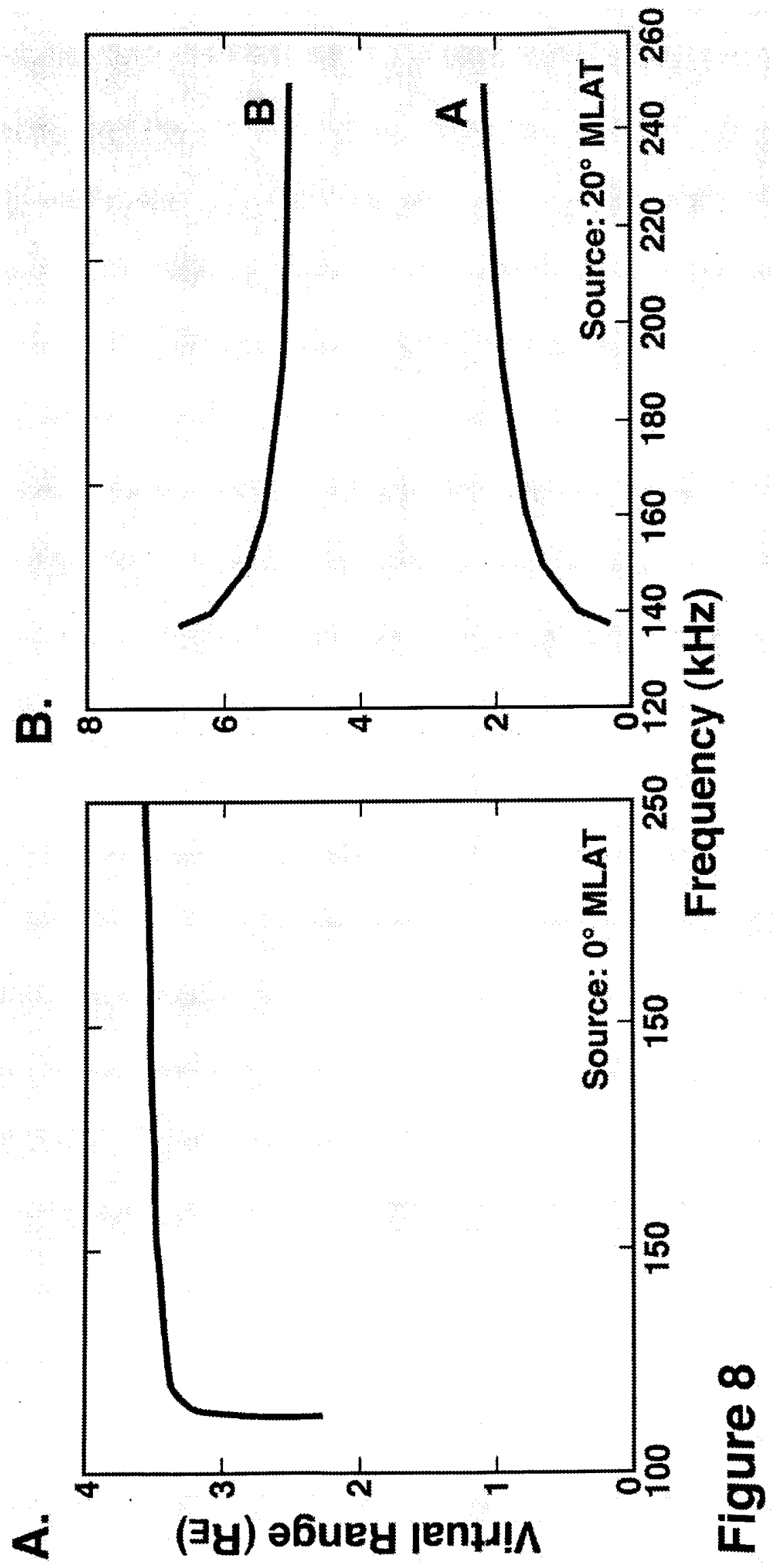




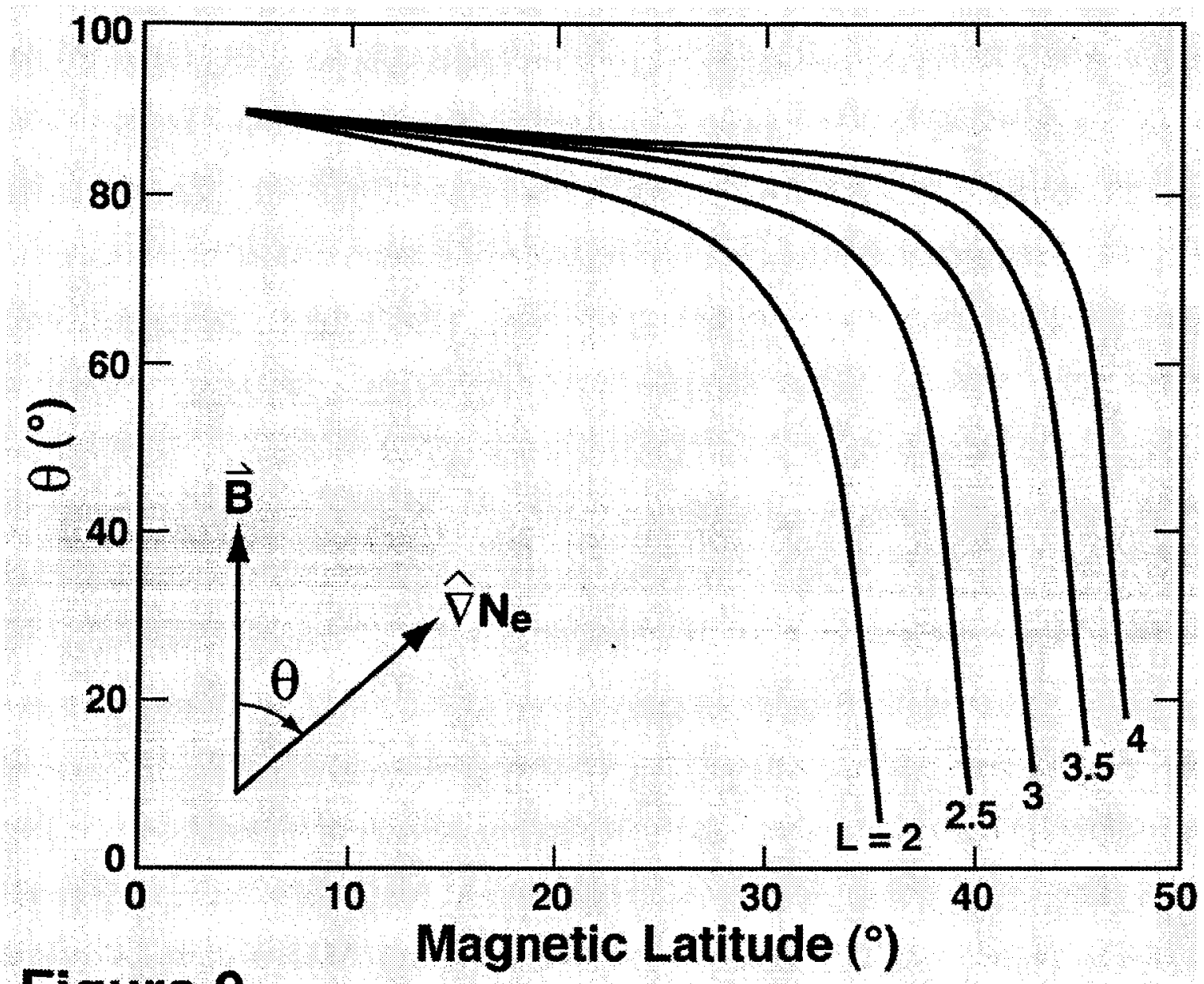

Figure 9 


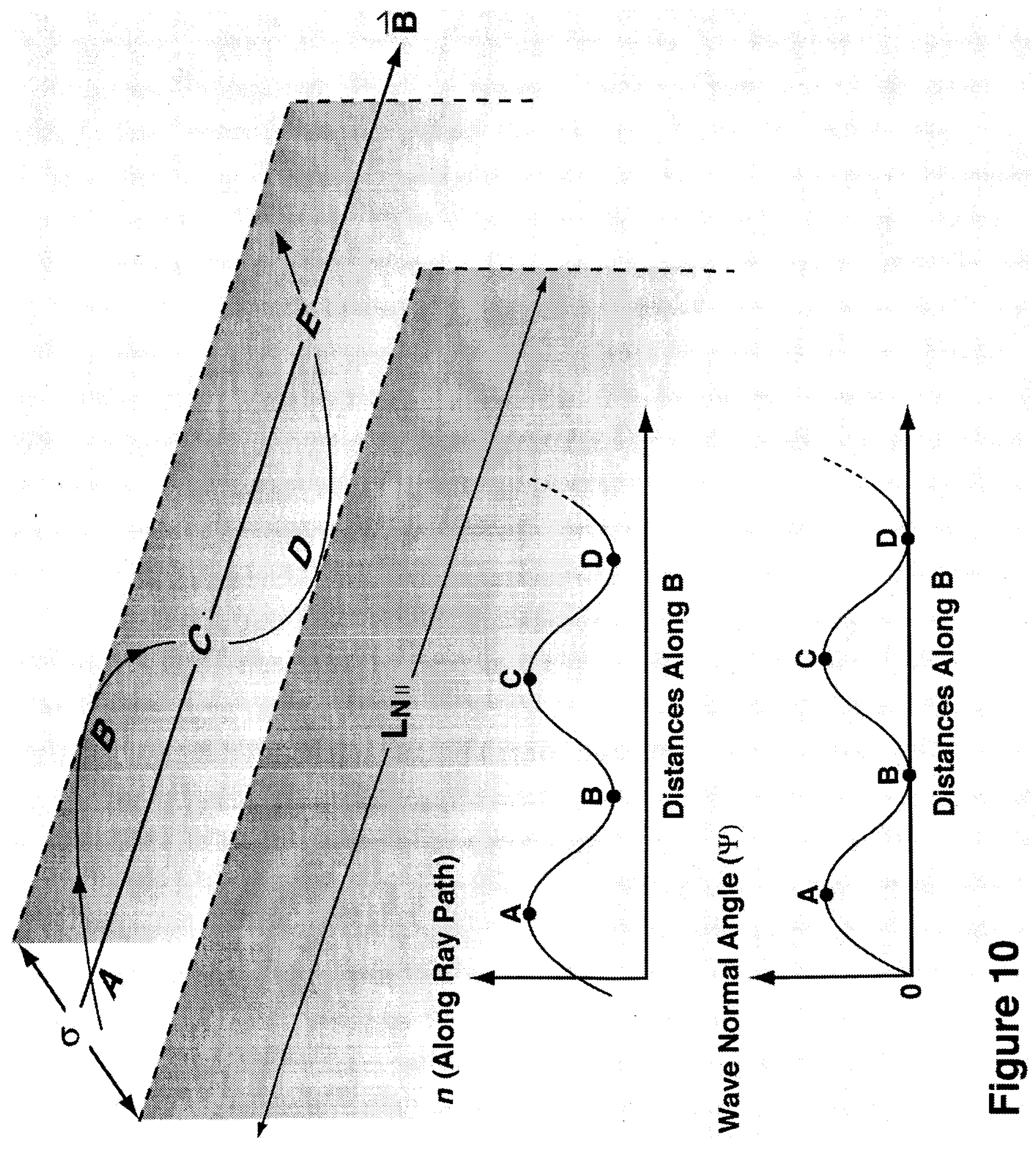

